\title{
MORFOLOGÍA ECOGRÁFICA DEL ÁPEX PROSTÁTICO: IMPLICACIONES EN SU DISECCIÓN DURANTE LA PROSTATECTOMÍA
}

\author{
F. HERRANZ AMO \\ Servicio de Urología. Hospital General Universitario Gregorio Marañón. Madrid.
}

Actas Urol Esp. 28 (6): 413-417, 2004

\section{RESUMEN}

MORFOLOGÍA ECOGRÁFICA DEL ÁPEX PROSTÁTICO: IMPLICACIONES EN SU DISECCIÓN DURANTE LA PROSTATECTOMÍA

El ápex prostático puede presentar diversas morfologias. Myers, basándose en piezas de prostatectomía, las clasificó en dos grupos: próstatas sin escotadura anterior (forma de "buñuelo") y con escotadura anterior (forma de "croissant"). El tiempo quirúrgico de la prostatectomía conocido como "disección apical”, debe de modificarse en función de la morfología del ápex prostático, para evitar la resección incompleta de la glándula. Mediante la ecografía transrectal, realizada en el mismo acto que la biopsia transrectal, es posible reconocer la morfología del ápex en cada glándula. Esto nos ayudaría a planificar la disección del ápex en cada caso de forma particular, evitando la sección incidental del labio posterior del ápex prostático.

PALABRAS CLAVE: Ápex prostático. Morfología. Ecografia transrectal de próstata. Disección apical. Prostatectomía radical.

\section{ABSTRACT \\ ULTRASOUND MORPHOLOGY OF PROSTATIC APEX: IMPLICATIONS FOR ITS DISSECTION IN PROSTATECTOMY}

The prostatic apex can present various morphologies. Myers, on the basis of prostatectomy specimens classified these into two main groups: prostates without an anterior dip (doughnunt shaped) and those with an anterior dip (croissant shaped). Surgical duration for prostatectomy known as "apical dissection" must be altered depending on the morphology of the prostatic apex, to prevent incomplete resection of the gland. With transrectal ultrasound performed during transrectal biopsy, the morphology of the apex can be established in each gland. This would help us to plan dissection of the apex in each specific case, avoiding unwanted dissection of the posterior lip of the prostatic apex.

KEY WORDS: Prostatic apex. Morphology. Prostate transrectal echography.

$\mathrm{L}$ a generalización de la determinación del PSA y de la ecografia transrectal de alta resolución en las consultas de Urología, está aumentando de forma muy importante los diagnósticos de cáncer de próstata ${ }^{1}$. Una de las consecuencias es el denominado "adelantamiento diagnóstico", los pacientes se diagnostican más jóvenes y en un estadio más precoz (migración del estadio). La mayoría de los pacientes que se diagnostican en la actualidad cumplen los requisitos para aplicarles un tratamiento con intención curativa. Esto ha aumentado en nuestros Centros el número de prostatectomías radicales que se hacen anualmente. 
La prostatectomía es una técnica quirúrgica de difícil realización que exige unos sólidos conocimientos anatómicos y una cierta destreza manual en su ejecución. El tiempo quirúrgico conocido como disección apical es especialmente difícil o delicado, ya que de la habilidad en su ejecución dependerán la continencia urinaria, la disfunción eréctil y en gran medida la existencia o no de márgenes quirúrgicos positivos ${ }^{2}$. Por lo tanto, el conocimiento previo a la cirugía de la morfología del ápex prostático es fundamental.

\section{MORFOLOGÍA ANATÓMICA DEL ÁPEX PROSTÁTICO}

Myers y cols. ${ }^{3}$ realizaron un estudio anatómico sobre 64 próstatas (30 especímenes obtenidos mediante prostatectomía y 34 de cadáver). La forma general de la glándula variaba desde cónica a esférica, presentando el ápex prostático diversas morfologías (Fig. 1), pero existía una característica en el ápex que según su existencia o ausencia podía dividir a todos los especímenes en dos grupos (Fig. 2). En 13 (43\%) de los especímenes conseguidos mediante prostatectomía la comisura anterior no se extendía distalmente hasta el ápex prostático, macroscópicamente estas próstatas parecían tener una muesca o una escotadura en su parte anterior. Las próstatas con muesca anterior tenian un volumen medio inferior (32 gr.) a las glándulas sin muesca anterior (42 gr.). La configuración del esfinter estriado externo dependía de la forma de la comisura anterior (Fig. 2). Por lo tanto, Myers $^{3,4}$ clasificó las próstatas según la morfología del ápex en dos clases: sin muesca o escotadura anterior a las que comparó con un "buñuelo" y las que presentaban muesca anterior que parecían tener forma de "croissant".
La sección de la uretra en el ápex prostático durante la prostatectomía en las glándulas sin muesca anterior es relativamente fácil. En cambio, en las próstatas que tienen escotadura anterior según Myers ${ }^{4}$, se pueden cometer 3 errores (Fig. 2): 1) al seccionar la uretra inmediatamente después de terminar la comisura anterior se puede seccionar el labio posterior del ápex, lo que implicaría una resección incompleta de la glándula; 2) si nos separamos mucho de la escotadura se resecará una parte importante del esfïnter estriado lo que repercutirá en la continencia del paciente; 3) en ocasiones la comisura es pequeña, fibrótica y corta, siendo fácil que durante su sección se penetre en la vejiga, lo que haría mucho más complicada la disección del ápex.

El conocimiento previo a la prostatectomía de la morfología del ápex prostático sería de gran importancia para la estrategia a seguir en la disección del ápex y la sección de la uretra en el ápex.

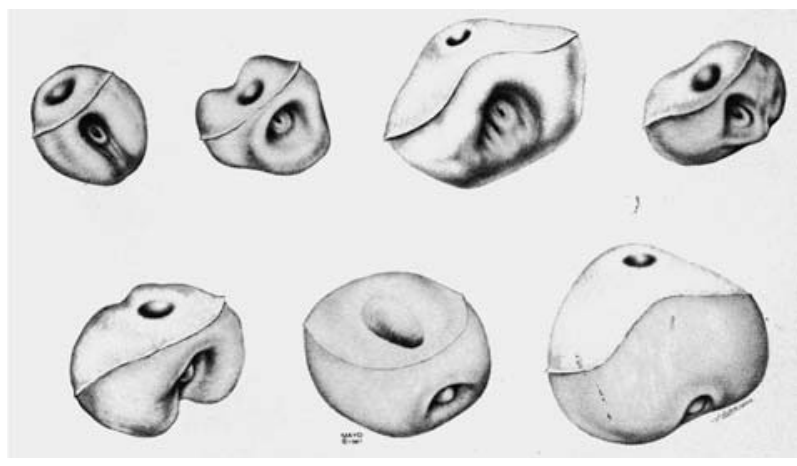

FIGURA 1. Diversidad en el tamaño y en la forma de la próstata. En la fila de arriba próstatas con distintas formas de escotadura anterior. En la fila de abajo próstatas sin escotadura anterior. La unión prostato-vesical se encuentra remarcada. Tomado de Myers (referencia bibliográfica $n^{\circ}$ 3).

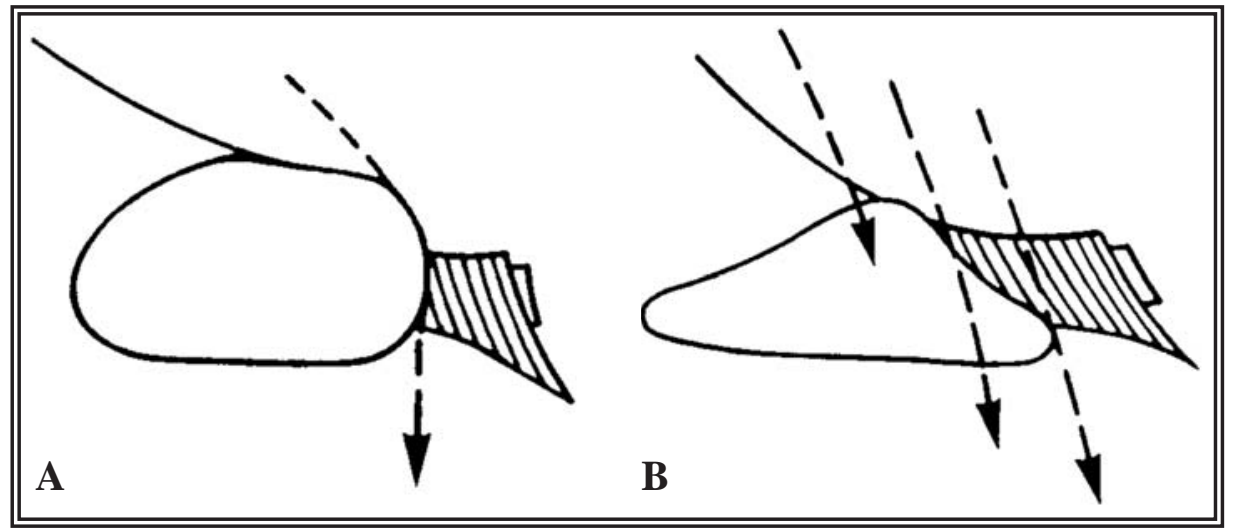

FIGURA 2. Las diversas formas de la Figura 1 se pueden agrupar en dos: A) sin escotadura anterior $y, B)$ con escotadura anterior. La flecha en $A$ indica la linea de sección de la uretra durante la prostatectomia. Las flechas en $B$ indican los posibles errores cometidos durante la sección de la uretra. Tomado de Myers (referencia bibliográfica no 4). 
Myers y cols. ${ }^{5}$ estudiaron la utilidad de la resonancia magnética en el conocimiento de la anatomía pélvica para su aplicación a la técnica de prostatectomía, pero en este artículo no se aportan datos sobre la morfología del ápex prostático. En octubre de 2003 se realizó una búsqueda bibliográfica en Medline con las siguientes palabras claves: "prostate anatomy", "prostate shape" y "transrectal ultrasound", no encontrando ningún artículo que analizara la morfología ecográfica del ápex prostático previo a la prostatectomía. Zisman y cols. ${ }^{6}$, utilizaron la ecografía transrectal intraoperatoria en 3 pacientes durante la prostatectomía, en uno de ellos después de la extracción de la pieza quirúrgica identificaron mediante la ecografía tejido apical residual que fue extirpado posteriormente.

\section{MORFOLOGÍA ECOGRÁFICA DEL ÁPEX PROSTÁTICO}

Las ecografías transrectales se realizaron con un ecógrafo Brüel \& Kjaer modelo 3535, con un transductor transrectal multiplanar a 7,5 Mhz, con canal de biopsia integrado tipo 8551, colocándose al paciente en posición de litotomía. Se realizaron cortes en longitudinal y en transverso a nivel del ápex prostático para identificar las estructuras anatómicas del mismo.

La morfología ecográfica del ápex prostático es muy variable y depende del grado de desarrollo de la hiperplasia benigna de próstata. Así en las próstatas pequeñas (Figs. 3 y 4) es difícil establecer claramente el límite anterior entre el ápex prostático y la uretra membranosa. Cuando comienza a desarrollarse la hiperplasia benigna el aumento del diámetro anteroposterior de la próstata favorece que se delimite de forma más clara la unión entre el ápex y la uretra membranosa (Figs. 5 y 6).

En algunas próstatas de pequeño tamaño se observa en los cortes ecográficos longitudinales a nivel del ápex que el labio posterior del mismo se prolonga por detrás de la uretra membranosa y entre ambas estructuras existe un área hiperecoica (grasa) (Figs. 7A y 7C). En los cortes ecográficos transversales a nivel de la unión del ápex
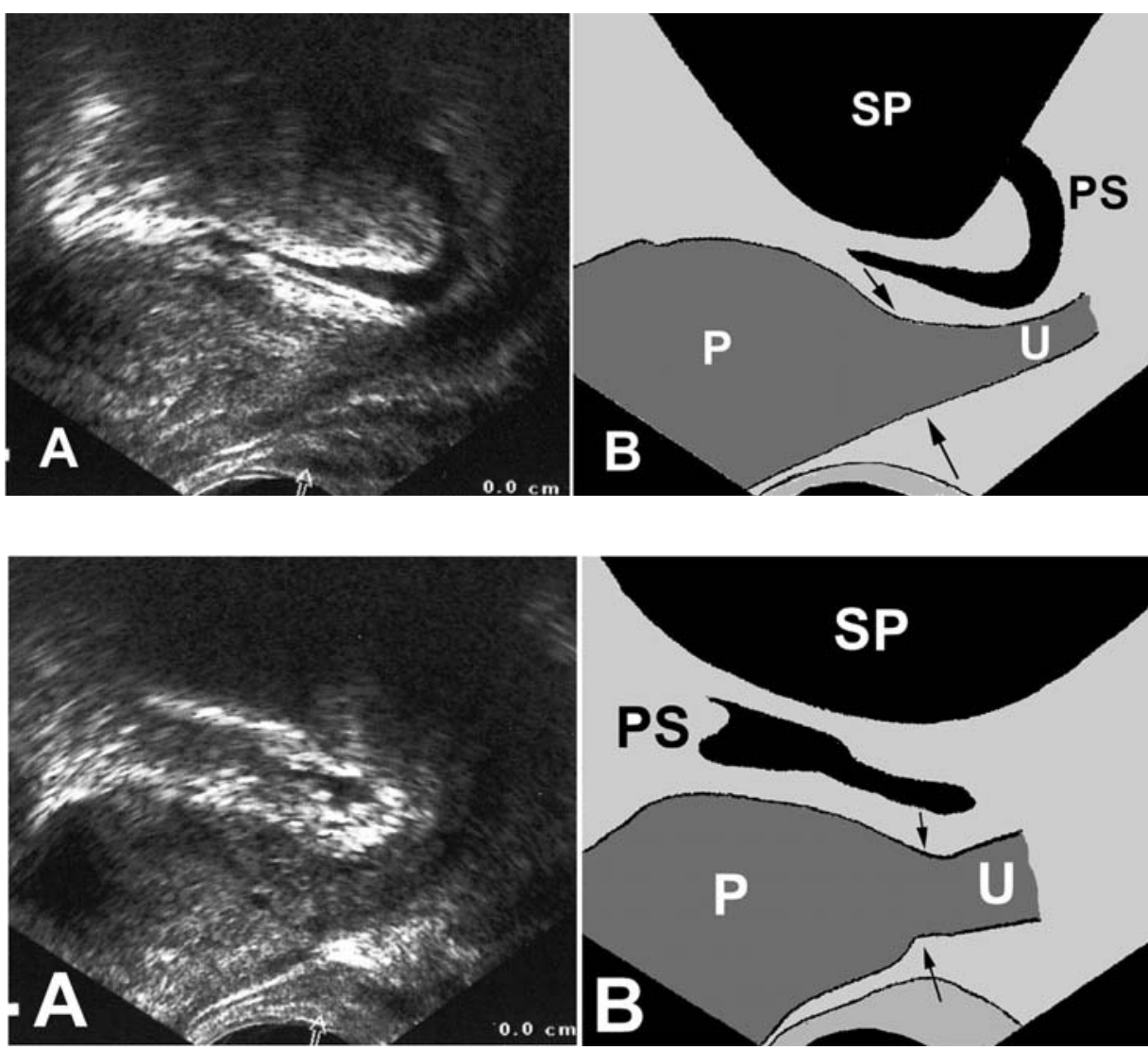

FIGURA 3. A) Ecografia transrectal, corte longitudinal a nivel del ápex prostático de una glándula de 21 cc. B) Esquema de la figura A. Las flechas señalan el límite del ápex- $P$ - próstata. U - uretra membranosa. PS - plexo venoso de Santorini. SP sombra del hueso pubis.

FIGURA 4. A) Ecografia transrectal, corte longitudinal a nivel del ápex prostático de una glándula de $20 \mathrm{cc}$. B) Esquema de la figura $A$. Las flechas señalan el limite del ápex. $P$ - próstata. U - uretra membranosa. PS - plexo venoso de Santorini. SP - sombra del hueso pubis. 

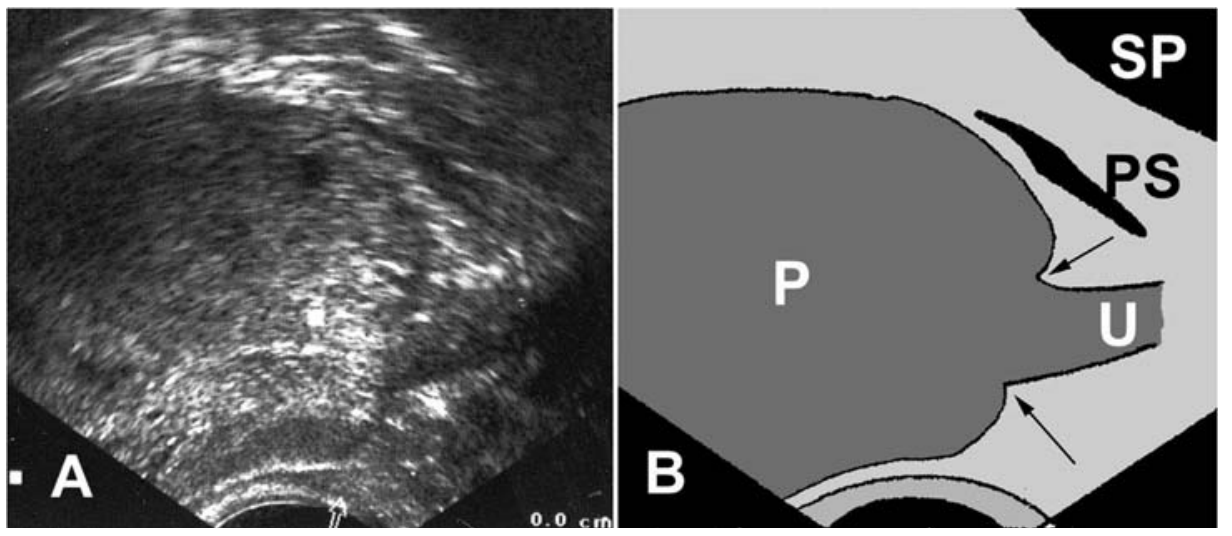

FIGURA 5. A) Ecografia transrectal, corte longitudinal a nivel del ápex prostático de una glándula de 71 cc. B) Esquema de la figura A. Las flechas señalan el limite del ápex. $P$ - próstata. U - uretra membranosa. PS - plexo venoso de Santorini. SP - sombra del hueso pubis.
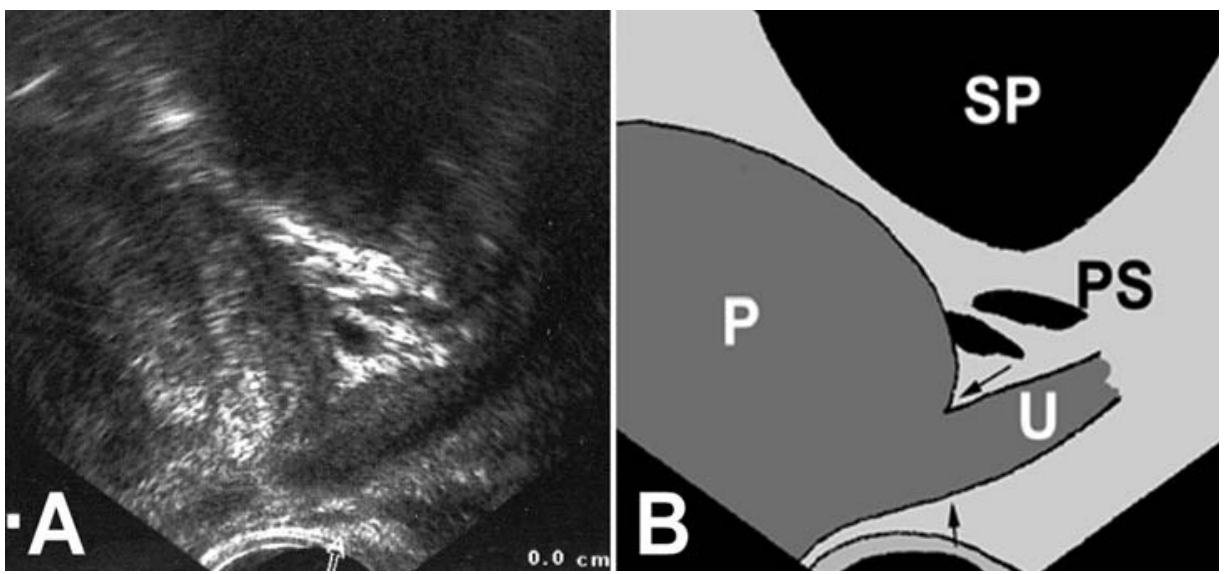

FIGURA 6. A) Ecografia trans rectal, corte longitudinal a nivel del ápex prostático de una glándula de 113 cc. B) Esquema de la figura $A$. Las flechas señalan el limite del ápex. $P$ - próstata. U - uretra membranosa. PS - plexo venoso de Santorini. SP - sombra del hueso pubis.
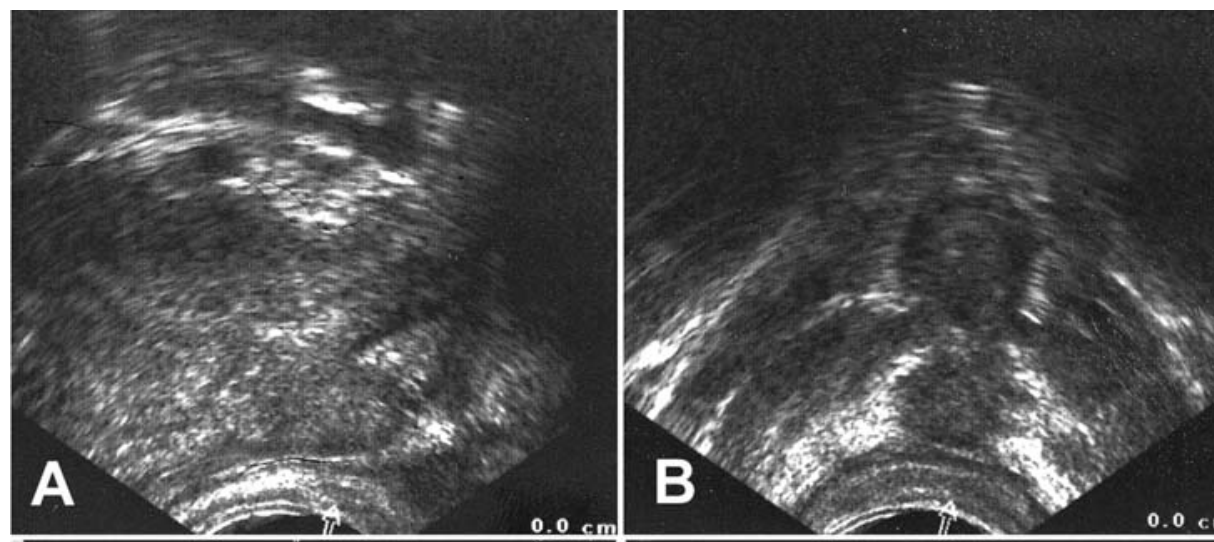

FIGURA 7. A) Ecografia transrectal, corte longitudinal a nivel del ápex prostático de una glándula de 24 cc con muesca o escotadura anterior. C) Esquema de la figura A, la línea blanca indica dónde se dio el corte transversal ecográfico. B) Corte transversal a nivel del ápex, se observa debajo de la uretra (U) el labio inferior del ápex prostático $(P)$ y entre ellos existe un plano graso. D) Esquema de la figura B. $P$ - próstata. $U$ - uretra membranosa. PS - plexo venoso de Santorini. SP - sombra del hueso pubis. EA - músculos elevadores del ano. 

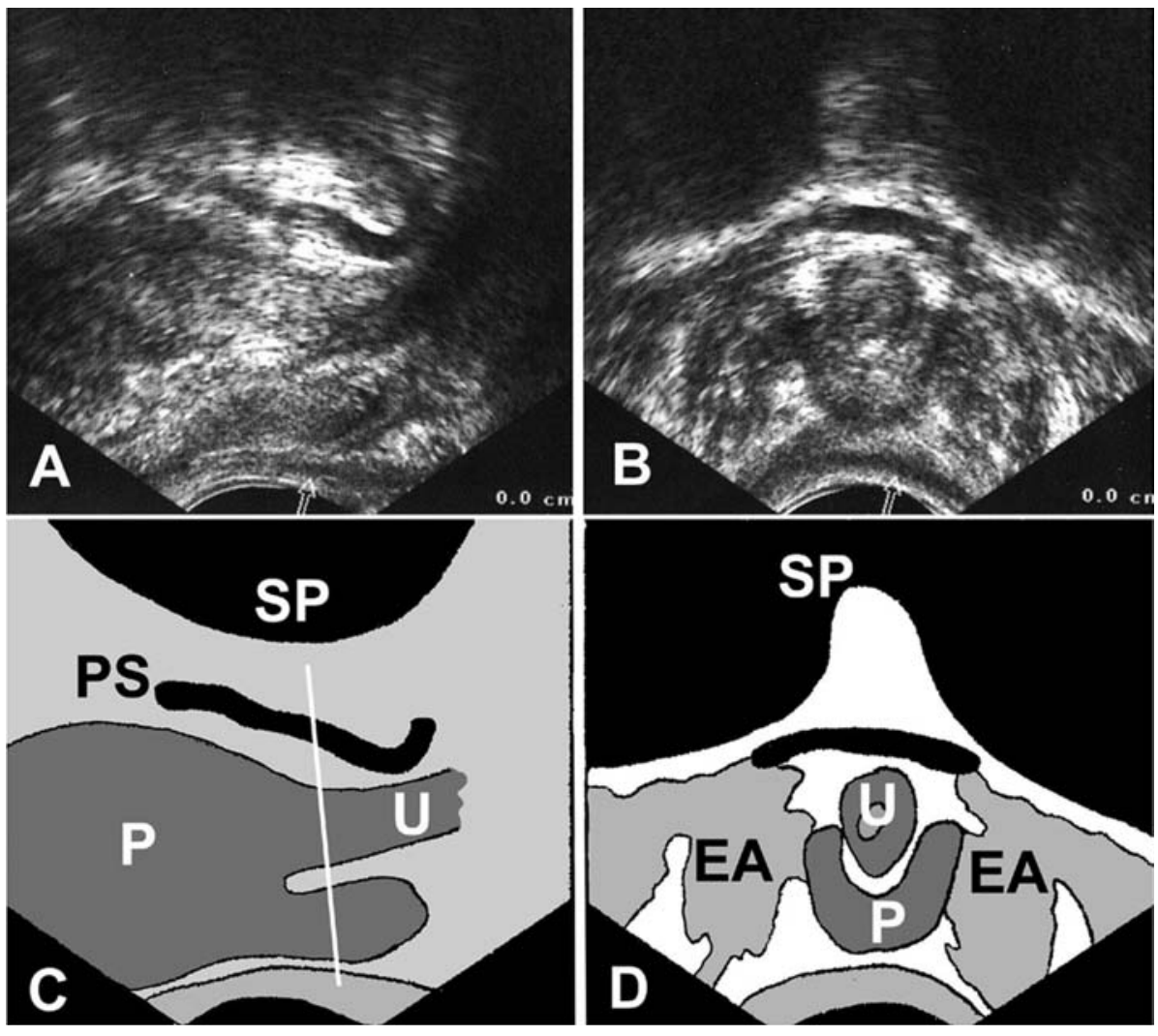

FIGURA 8. A) Ecografia transrectal corte longitudinal a nivel del ápex prostático de una glándula de 18 cc cn muesca o escotadura anterior; C) Esquema de la figura $A$, la linea blanca indica dónde se dio el corte transversal ecográfico; B) Corte transversal a nivel del ápex, se observa debajo de la uretra (U) el labio inferior del ápex prostático (P) y entre ellos existe un plano graso; D) Esquema de la figura $B$. $P$ - próstata. U - uretra membranosa. PS - plexo venoso de Santorini. SP - sombra del hueso pubis. EA - músculos elevadores del ano.

con la uretra membranosa, se observa el labio posterior del ápex prostático por debajo de la uretra separados por un plano graso (Figs. 7B y 7D). Cuando el labio posterior del ápex es mucho más prominente (Figs. 8A y 8C), en las secciones transversales se observa como el ápex además de prolongarse por detrás de la uretra, abraza a ambas caras laterales de la misma (Figs. 8B y 8D). Esta morfología ecográfica se corresponde con lo que Myers y cols. ${ }^{3}$ denominaron como próstatas con muesca anterior o en "croissant".

Mediante secciones ecográficas longitudinales y transversales a nivel del ápex prostático es posible identificar a los pacientes que tienen una morfología del ápex prostático con muesca, escotadura anterior o próstata en "croissant". El conocimiento de esta morfología del ápex previo a la prostatectomía evitará la sección inadvertida del labio posterior del ápex, lo que originaría una resección incompleta de la glándula con márgenes incidentales en el supuesto de que no existiera tumor en el ápex o con tumor residual en el peor de los casos.

\section{REFERENCIAS}

1. HERRANZ AMO F, ARIAS FÚNEZ F, ARRIZABALAGA MORENO M y cols.: El cáncer de próstata en la Comunidad de Madrid en el año 2000. I - Incidencia. Actas Urol Esp 2003; 27: 323-334.

2. GILLITZER R, THÜROFF JW.: Technical advances in radical retropubic prostatectomy techniques for avoiding complications. Part I: apical dissection. BJU International 2003; 92: 172-177.

3. MYERS RP, GOELLNER JR, CAHILL DR.: Prostate shape, external striated urethral sphincter and radical prostatectomy: the apical dissection. J Urol 1987; 183: 543-550.

4. MYERS RP.: Male urethral sphincter anatomy and radical prostatectomy. Urol Clin Nort Am 1991; 18 (2): 211-227.

5. MYERS RO, CAHILL DR, DEVINE RM, KING BF.: Anatomy of radical prostatectomy as defined by magnetic resonance imaging. J Urol 1998; 159: 2148-2158.

6. ZISMAN A, STRAUSS S, SIEGEL YI, MANOR H, LINDER A.: Transrectal ultrasonographically assisted radical retropubic prostatectomy. $J$ Ultrasound Med 1997; 16: 777-782.

Dr. F. Herranz Amo

C/ Doctor Esquerdo, 155 $5^{\mathrm{a}}-7^{\text {o }} 3$

28007 Madrid

(Trabajo recibido el 16 diciembre de 2003) 